\title{
Implants dentaires et valvulopathies : pour une évolution des recommandations
}

\author{
Hélène Meurisse ${ }^{1,{ }^{*}}$, Stéphane Milliez ${ }^{1}$, Pascal Gueret ${ }^{2}$, Sophie-Myriam Dridi ${ }^{1}$, \\ Bruno Gogly ${ }^{1}$, Frédérick Gaultier ${ }^{1}$ \\ ${ }^{1}$ Groupe hospitalier Mondor-Chenevier, Service d'Odontologie, Université Paris Descartes, Paris, France \\ ${ }^{2}$ Groupe hospitalier Mondor-Chenevier, Service de Cardiologie, Université Paris 12, Paris, France
}

(Reçu le 31 mai 2012, accepté le 20 juin 2012)

Mots clés :

implants /

valvulopathies

Key words: implants / valvular heart diseases

\begin{abstract}
Résumé - L'AFSSAPS a publié de nouvelles recommandations en septembre 2011 relatives à l'antibioprophylaxie en odontologie. Les patients appartenant à l'ancien groupe à risque modéré d'endocardite infectieuse (insuffisance mitrale, insuffisance aortique, rétrécissement aortique...) intègrent désormais le groupe de la population générale et devraient donc être, à ce titre, traités de façon identique. Cependant, au vu du risque de l'évolution de ces pathologies, il parait nécessaire de poser des limites au traitement implantaire afin de prévenir le risque infectieux.
\end{abstract}

\begin{abstract}
Dental implants and valvular heart diseases: for an evolution of recommendations. AFSSAPS published in September 2011 new recommendations in odontology about antibiotics prophylaxis. The patients belonging to the former moderate risk group of infectious endocarditis (mitral and aortic insufficiencies, aortic stenosis...) are now considered as the general population and should thus be treated in the same way. However, considering the risk correlated to the evolution of these pathologies, it is necessary to set limits for the implant treatment in order to prevent any infection risk.
\end{abstract}

Les nouvelles recommandations de l'AFSSAPS, parues en septembre 2011, redéfinissent les indications de la prescription des antibiotiques dans la pratique bucco-dentaire [1].

La notion de risque $A$ et $B$, et de groupes $A$ et $B$ est réactualisée et la population est divisée en 3 groupes :

- la population générale ;

- les patients immunodéprimés, ;

- les patients à haut risque d'endocardite infectieuse (prothèse valvulaire, antécédent d'endocardite infectieuse, cardiopathie congénitale cyanogène).

Selon ces recommandations, l'implantologie est autorisée chez tous les patients de la population générale et n'est pas contre-indiquée chez les patients immunodéprimés. Les patients atteints de valvulopathies, notamment les patients ayant une insuffisance mitrale ou aortique (appartenant à l'ancien groupe à « risque modéré » d'endocardite), sont donc susceptibles d'avoir recours à l'implantologie. Pour les patients atteints de valvulopathies, notamment de valvulopathies aortiques, l'implantologie n'est pas contre-indiquée mais n'est pas clairement autorisée non plus. Les nouvelles recommandations n'apportent pas plus de précisions sur les possibilités de poser des implants chez les patients qui ne sont plus considérés à risque d'endocardite infectieuse.

À ce titre, il semble nécessaire de suggérer quelques restrictions ou tout au moins d'établir des règles encadrant la pose d'implants chez ces patients.

\section{Evolution des valvulopathies}

La prévalence des cardiopathies valvulaires est de $2 \%$ dans les pays occidentaux avec une augmentation notable avec l'âge ; 10 à $15 \%$ des sujets de plus de 75 ans sont porteurs d'une cardiopathie valvulaire [2]. Le vieillissement de la population entraine surtout une augmentation notable des valvulopathies dystrophiques (insuffisance mitrale) et dégénératives

\footnotetext{
*Correspondance : helene_meurisse@yahoo.fr
} 
Tableau I. Résultats de l'étude de Roccuzzo et al. [8]. Table I. Data of the Roccuzzo et al. study [8].

\begin{tabular}{ccc}
\hline & Taux de survie implantaire & $\%$ de sites présentant une perte osseuse $>3 \mathrm{~mm}$ \\
Patients sains & $96,6 \%$ & $4,7 \%$ \\
Parodontite modérée & $92,8 \%$ & $11,2 \%$ \\
Parodontite sévère & $90 \%$ & $15,1 \%$ \\
\hline
\end{tabular}

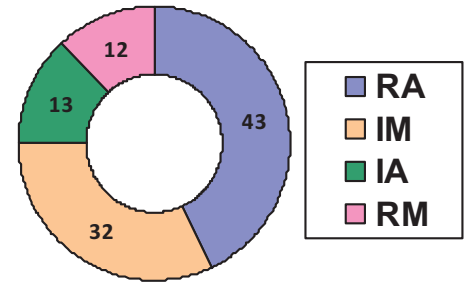

Fig. 1. Répartition des valvulopathies en Europe. (RA : rétrécissement aortique, IM : insuffisance mitrale, IA : insuffisance aortique, $\mathrm{RM}$ : rétrécissement mitral).

Fig. 1. Distribution of valvular heart diseases in Europa. (RA: aortic stenosis, IM: mitral insufficiency, IA: aortic insufficiency, RM: mitral stenosis).

(rétrécissement aortique) (Fig. 1). Leur traitement consiste soit à réparer la valve (« plastie » mitrale, plus rarement aortique), soit à mettre en place une prothèse artificielle si la réparation n'est pas possible. En cas de rétrécissement aortique serré, le remplacement valvulaire est le seul traitement à proposer dès que le patient est symptomatique.

Selon l'évolution, le patient âgé atteint d'une valvulopathie peut devenir porteur d'une prothèse valvulaire et, par la même occasion, un patient du groupe à haut risque d'endocardite infectieuse. Or, d'après les recommandations de l'AFSSAPS, les traitements implantaires mais surtout le traitement des périimplantites sont contre-indiqués pour les patients à haut risque d'endocardite.

La sévérité et le risque d'évolution de la pathologie cardiaque est donc à prendre en compte lors de la décision d'un traitement implantaire ; ce risque est à évaluer en collaboration avec le cardiologue du patient selon les critères coût/ bénéfice/sécurité.

\section{Tabac et péri-implantite}

La prévalence des péri-implantites varie beaucoup selon les articles car les auteurs n'ont pas tous les mêmes critères d'inclusion (critères radiologiques, de sondage ....) [3]. La périimplantite associe, en général, une inflammation gingivale et une perte osseuse avec exposition des premières spires de l'implant. Cette complication secondaire ou tardive de l'implant ostéo-intégré représente un foyer infectieux potentiel aigu ou chronique.
Des études publiées récemment insiste sur l'influence néfaste du tabac sur la longévité des implants et sur la relation directe entre tabac et survenue de péri-implantites. En 1997 déjà, dans une étude portant sur 50 patients, Lindquist et al. avaient montré qu'il existait une corrélation entre la perte osseuse péri-implantaire et la quantité de cigarettes consommées [4]. En 2009, à partir d'une revue de littérature, Liza met en évidence que le risque de péri-implantite est accru chez les fumeurs par rapport aux non fumeurs (avec un odd ratio de 3,6 à 4,6 ) et qu'il est plus accru encore si le fumeur présente une parodontite traitée [5]. En 2011, Rodriguez-Argueta et al. ont publié une étude rétrospective portant sur les complications postopératoires chez 295 patients : le patient fumeur développe significativement plus de péri-implantites $(9,3 \%)$ que le patient non fumeur $(5,3 \%)$ [7].

\section{Maladie parodontale et péri-implantite}

La maladie parodontale peut être définie comme une accumulation de bactéries pathogènes qui crée un déséquilibre de la flore buccale et entraîne une dégradation du parodonte (gencive, os...). Après le traitement, ou plutôt la stabilisation, le patient se doit de maintenir une hygiène buccale irréprochable sous peine d'une récidive.

Plusieurs études se sont donc intéressées à la relation entre un antécédent de parodontite et le développement d'une périimplantite $[10,11]$. Une revue de littérature réalisée en 2009 met en évidence la difficulté de regrouper les études, en raison des critères utilisés (parodontites sévères, modérées... avec ou sans tabac) mais rapporte surtout que les patients ayant un antécédent de parodontite ont davantage de risque de développer une péri-implantite [9].

L'étude prospective de Roccuzzo et al. comportant 112 patients suivis sur 10 ans démontre qu'il y a une corrélation entre un antécédent de parodontite et le nombre de sites implantaires ayant une perte osseuse supérieure à $3 \mathrm{~mm}$ (Tab. I) [8]. La parodontite représente donc un indicateur potentiel du risque de développer une péri-implantite.

\section{Conditions favorisant le succès implantaire : contexte local}

La présence d'un défaut osseux dans la zone à implanter représente une difficulté supplémentaire et un échec potentiel 
Tableau II. Indications implantaires en présence d'une valvulopathie. Table II. Implant indications in front of valvular heart diseases.

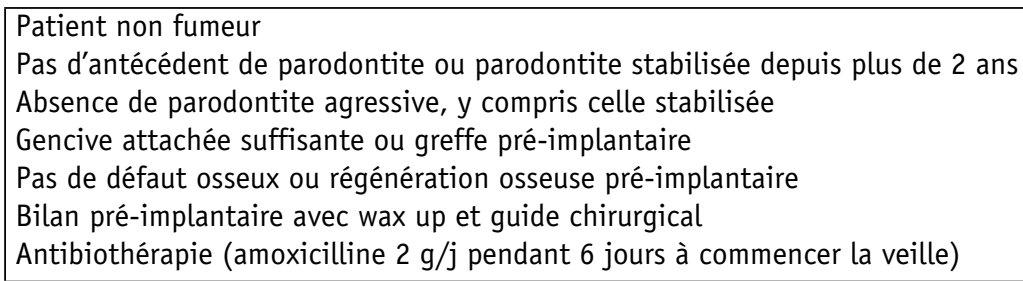

supplémentaire, ainsi qu'une augmentation du risque de survenue d'une péri-implantite à terme, si tout ou partie de l'implant est exposé hors de l'os lors de la pose. Il est donc absolument nécessaire de dissocier les techniques de reconstruction osseuse et la pose de l'implant.

L'association des deux techniques chirurgicales dans le même temps opératoire est à proscrire

De la même manière, le site implantaire ne doit pas comporter de défaut de gencive attachée. Si tel est le cas, il est nécessaire de gérer le déficit tissulaire par des techniques préimplantaires muco-gingivales. La mise en place de l'implant se fait en deux temps chirurgicaux avec des implants enfouis dont le délai de mise en nourrice est de 2-3 mois à la mandibule et de 3-4 mois au maxillaire.

La réalisation d'un wax up et d'un guide chirurgical permet de réaliser une mise en fonction simple, avec un axe implantaire idéal et une émergence implantaire située au sein des tissus kératinisés. Ce protocole permet donc de réduire considérablement les risques iatrogènes de survenue de péri-implantite.

Il a été démontré que l'on obtenait un meilleur taux de succès avec la prescription d'une antibioprophylaxie $(2 \mathrm{~g}, 1$ heure avant l'intervention) qu'en l'absence d'antibioprophylaxie [12]. Dans notre pratique, nous conseillons une antibiothérapie commencée la veille de l'intervention et poursuivie pendant 6 jours à raison de $2 \mathrm{~g}$ d'amoxicilline par jour.

\section{Conclusion}

Selon les nouvelles recommandations de l'AFSSAPS éditées en septembre 2011, les patients atteints de valvulopathies font partie intégrante de la population générale et, à ce titre, sont donc éligibles aux mêmes thérapeutiques odontologiques, notamment implantaires.

Compte tenu des échecs observés dans la population générale, il est impératif de prendre en considération les risques de péri-implantite chez les patients atteints d'une valvulopathie. Il faudra donc exclure les patients fumeurs et ayant eu une maladie parodontale (Tab. II) [6]. Les critères locaux (volume osseux alvéolaire résiduel et présence d'une gencive attachée) sont deux critères cliniques majeurs dont l'évaluation et la reconstruction éventuelle s'avèrent nécessaire pour optimiser le traitement implantaire. Une antibiothérapie commencée la veille de l'intervention chirurgicale est recommandée (Tab. II).
Enfin, une étroite collaboration avec le cardiologue du patient est indispensable pour dèterminer l'éligibilité réelle du patient pour une thérapeutique implantaire en fonction de la sévérité de sa valvulopathie et son potentiel évolutif.

\section{Conflits d'intérêt : aucun}

\section{Références}

1. AFSSAPS. Prescription des antibiotiques en pratique buccodentaire. Recommandations 2011.

2. HAS. Cardiopathies valvulaires et congénitales graves chez l'adulte. Guide médecin, 2008.

3. Zitzmann NU, Berglundh T. Definition and prevalence of periimplant diseases. J Clin Periodontol 2008;35(Suppl.8):286-91.

4. Lindquist LW, Carlsson GE, Jemt T. Association between marginal bone loss around osseointegrated mandibular implants and smoking habits: a 10-year follow-up study. J Dent Res 1997;76:1667-74.

5. Heitz-Mayfield LJ, Huynh-Ba G. History of treated periodontitis and smoking as risks for implant therapy. Int J Oral Maxillofac Implants 2009;24(Suppl):39-68.

6. Heitz-Mayfield LJ. Peri-implant diseases: diagnosis and risk indicators. J Clin Periodontol 2008;35(Suppl.8):292-304.

7. Rodriguez-Argueta OF, Figueiredo R, Valmaseda-Castellon E, GayEscoda C. Postoperative complications in smoking patients treated with implants: a retrospective study. J Oral Maxillofac Surg 2011;69:2152-7.

8. Roccuzzo M, De Angelis N, Bonino L, Aglietta M. Ten-year results of a three arms prospective cohort study on implants in periodontally compromised patients. Part 1: implant loss and radiographic bone loss. Clin Oral Impl Res 2010;21:490-6.

9. Renvert S, Persson GR. Periodontitis as a potential risk factor for peri-implantitis. J Clin Periodontol 2009;36(Suppl.10):9-14.

10. Ong CT, Ivanovski S, Needleman IG, Retzepi M, Moles DR, Tonetti MS, Donos N. Systematic review of implant outcomes in treated periodontitis subjects. J Clin Periodontol 2008;35:438-62.

11. Safii SH, Palmer RM, Wilson RF. Risk of implant failure and marginal bone loss in subjects with a history of periodontitis: a systematic review and meta-analysis. Clin Implant Dent Relat Res 2010;12:165-74.

12. Meurisse H, Gueret P, Dridi SM, Ferré F, Cabecadas AS, Gaultier F. Valvulopathies et implants dentaires : vers une évolution des recommandations. Consensus Cardio 2010;62: 21-4. 